\title{
Preterm Prelabor Rupture of Membranes and Fetal Survival after Minimally Invasive Fetal Surgery: A Systematic Review of the Literature
}

\author{
Veronika Beck ${ }^{\mathrm{a}-\mathrm{c}}$ Paul Lewi ${ }^{\mathrm{a}}$ Leonardo Gucciardo ${ }^{\mathrm{a}, \mathrm{b}}$ Roland Devlieger ${ }^{\mathrm{a}}$ \\ a Division of Woman and Child, Department of Obstetrics and Gynaecology, University Hospital Gasthuisberg, \\ and ${ }^{b}$ Centre for Surgical Technologies, Faculty of Medicine, Katholieke Universiteit Leuven, Leuven, Belgium; \\ 'Universitätsfrauenklinik Tübingen, Tübingen, Germany
}

\section{Key Words}

Acardiac twin • Fetal therapy $\cdot$ Laser coagulation •

Obstructive uropathy - Premature rupture of membrane •

Twin-twin transfusion syndrome $\cdot$ Lower urinary tract obstruction - Twin-reversed arterial perfusion - Laser

ablation $\cdot$ Fetal shunt $\cdot$ Cord occlusion

\begin{abstract}
Objective: latrogenic preterm prelabor rupture of membranes (iPPROM; $<37$ weeks of gestation) is a major complication of fetal surgery. Little information is available about risk factors and incidence. Methods: We systematically reviewed reported iPPROM rates, gestational age at delivery and fetal survival after representative minimally invasive antenatal procedures. Results: A total of 1,146, 36 and 194 cases with mean iPPROM rates of 27,31 and $26 \%$ were included for placental laser in twin-twin transfusion syndrome, shunting in lower urinary tract obstruction and interventions for twin-reversed arterial perfusion, respectively. In the statistical analysis, the maximum diameter of the instrument predicted IPPROM rate and was significantly related to gestational age at birth as well as fetal survival. Information on duration of the respective procedures was scarce and did not allow for meaningful analysis. Conclusions: iPPROM occurs
\end{abstract}

in about $30 \%$ of cases treated by minimally invasive fetal surgery. The maximum diameter of the instrument explains iPPROM rate, gestational age at birth and fetal survival. Great variations in the reporting of IPPROM make data analysis difficult.

Copyright $\odot 2011$ S. Karger AG, Basel

\section{Background}

Minimally invasive fetal surgery nowadays is performed for a selected number of fetal conditions. The procedures are mostly carried out under ultrasound guidance and can be divided into two categories. First, the relatively well-established 'needle techniques' as for instance intrauterine blood sampling and transfusion in the case of fetal anemia or fetal shunting for lower urinary tract obstructions (LUTO) and pleural effusions, in which a sharp needle of various diameters is introduced into the uterine cavity under ultrasound guidance. Secondly, the fetoscopic procedures that are of-

This work was conducted at the Katholieke Universiteit Leuven, Leuven, Belgium.

\section{KARGER}

Fax +41613061234 E-Mail karger@karger.ch www.karger.com
Roland Devlieger, $\mathrm{MD}, \mathrm{PhD}$

Division of Woman and Child

Department of Obstetrics and Gynaecology, University Hospitals Leuven

Herestraat 49, BE-3000 Leuven (Belgium)

Tel. +32 163322 11, E-Mail roland.devlieger@uzleuven.be 
fered in an increasing number of centers worldwide. Here, ultrasound guidance is combined with a direct fetoscopic view to perform the intervention. The most common indication for fetoscopic surgery is the laser ablation of placental anastomoses in twin-twin transfusion syndrome (TTTS). Placental laser surgery was proven to be superior to amnioreduction in a randomized trial [1] and, hence, is the present standard of care. Another relatively prevalent fetoscopic procedure is endoluminal tracheal occlusion for congenital diaphragmatic hernia, although the latter is still being investigated in a randomized trial (www.totaltrial.org). In monochorionic pregnancies complicated by twin-reversed arterial perfusion (TRAP) sequence, different antenatal intervention strategies have been demonstrated to be effective including ultrasound-guided radiofrequency ablation (RFA), bipolar cord occlusion as well as fetoscopic laser surgery [2-6].

Although iatrogenic preterm prelabor rupture of membranes (iPPROM) seems to be less frequent than in open fetal surgery [7], it remains the most important complication of minimally invasive fetal procedures. Potential sequelae of PPROM include oligohydramnios-related pulmonary hypoplasia, chorioamnionitis and preterm delivery resulting in a considerable morbidity and mortality of affected infants. Regarding the incidence of iPPROM, reported numbers vary significantly amongst authors, even when the same technique is applied to comparable pathologies $[8,9]$. Little is known about underlying pathomechanisms and potential influencing factors of iPPROM. Parameters that have been suggested to impact iPPROM rates are the diameter of the surgical instrument and the number of entries to the uterine cavity amongst others $[10,11]$. However, no certainty about these potential risk factors exists.

As the rate of iPPROM and subsequent prematurity largely determine the success of any invasive fetal intervention, it is important to obtain more information on incidence and pathophysiology of iPPROM. For this reason, we undertook a systematic review of the literature on different fetal interventions and report on iPPROM rates in relation to the characteristics of the respective publications.

\section{Data Sources}

Our objective was to study iPPROM after minimally invasive fetal surgery in humans. As not all different procedures could be studied, we selected relevant established interventions with a broad spectrum in the diameter of uterine access: fetoscopic sur- gery for TTTS, percutaneous bladder shunting for LUTO and different antenatal interventions for TRAP. We reviewed all published studies and case reports in peer-reviewed journals until April, 2010. Relevant literature was identified using the electronic bibliographic databases PubMed and ISI web of science. In addition, a manual search was performed from the reference lists of all identified articles. The keywords used in the electronic search for fetoscopic surgery for TTTS were PROM, amniotic leak, amniorrhexis and membrane rupture in combination with twin-transfusion syndrome and $f(o)$ etoscopy or $f(o)$ etoscopic surgery. For LUTO we applied the search terms LUTO, lower urinary tract obstruction, bladder, megacystis, urethral valve, hydronephrosis in combination with $f(o)$ etal shunt and PROM, amniotic leak, amniorrhexis and membrane rupture. Keywords for TRAP-related interventions were TRAP, twin-reversed perfusion, acardiac as well as pump twin in combination with $f(o)$ etal surgery, $f(o)$ etal intervention, $f(o)$ etal procedure, RFA, radiofrequency ablation, cord occlusion, laser and PROM, amniotic leak, amniorrhexis and membrane rupture.

Language of eligible articles was restricted to English. Cases reported only as abstracts, single case reports or double publications were not included. We subsequently excluded all papers that had used plugs or amniopatch to seal the membrane defect. Further, we excluded papers, if during the same pregnancy prior to the studied intervention a second intrauterine procedure other than a mere needle procedure had been performed. Cases in which no fetal procedure was done were also excluded. Furthermore, we accepted articles for inclusion only if they minimally contained the following information: gestational age at intervention, delivery and iPPROM; diameter of the instrument used; number of accesses to the uterine cavity made, and number or percentage with iPPROM of all cases reported.

The primary outcome studied was iPPROM rate. Secondary outcomes were gestational age at birth and fetal survival.

The identified studies were independently reviewed by two of the authors (R.D. and V.B.). Disagreements regarding inclusion of a specific article were resolved by consensus. Only one criterium for exclusion is specified per article.

Differences between the Protocol and the Review

Because of the small number of papers ( 4 articles on 16 cases meeting inclusion criteria) detected for LUTO after a literature search with the specified combinations of 3 keywords, we omitted PROM, amniotic leak, amniorrhexis and membrane rupture in a second search.

We initially attempted to include only papers that would specify a gestational age at iPPROM. However, this was the case in only $\sim 50 \%$ of detected articles, which we considered an important finding. Therefore, we did not exclude these papers.

\section{Statistical Analyses}

One-way analysis of variance (ANOVA) of categorical variables and bivariate as well as a stepwise multivariate regression analysis of continuous variables were performed on the pooled data of the 3 interventions studied. iPPROM rate was only considered if it was reported until 37 weeks for all cases. Median and mean values specified in the articles were not distinguished, nor was the follow-up time for survival rates. Repeated shunt interventions for LUTO were included in the calculation of the gestational age at therapy. Analyses were carried out using statistical 
analysis software (JMP 8; SAS, Cary, N.C., USA). p values $<0.05$ were considered statistically significant. Data are presented as mean or median with range or standard deviation as provided by the original publications.

This systematic review was accomplished according to the guidelines of the Cochrane Handbook for Systematic Reviews of Interventions (version 5.0.2, last update September, 2009; www. cochrane-handbook.org).

\section{Results}

\section{Placental Laser Ablation for TTTS}

Applying our search criteria for TTTS resulted in 64 detected articles, 36 of which were retrieved from references. Nineteen publications were excluded in the first (V.B.), another 33 (V.B. and R.D.) during the second screening. Reasons for exclusion were: insufficient information (18), review (10), membrane sealing (8), double report (7), single case report (3), unrelated topic (3), animal study (1), language (1) and editorial (1). This left 12 publications on 1,146 cases for inclusion in our review (table 1a). Six of these publications report on 6-33 cases, 5 articles are on larger case series $(n=72-152)$ and 1 comprises a big series of 438 cases. We identified 2 randomized controlled trials $[1,12]$, both of which were excluded in favor of more recent publications on enlarged case series of the same research groups $[13,14]$.

Laser surgery for TTTS was carried out via 1-2 ports with a mean maximal diameter of $3.3(2.2-4) \mathrm{mm}$. Mean gestational age was $21(15-28)$ and 31 (18-41) weeks at therapy and delivery, respectively. iPPROM occurred in an average of $27 \%(11-50 \%)$ of cases, however, was not specified until 37 weeks in 5/12 publications (42\%). Instead iPPROM was then given for a limited number of weeks or a subset of fetuses: iPPROM within 2-3 weeks after the intervention ( 3 articles), until 36 weeks (1), in viable infants (1) and iPPROM associated with oligohydramnios (1). Similar information was found for the gestational age at iPPROM: 3 articles did not report a gestational age and 6 gave relative information. In the remaining 3 articles, iPPROM occurred at an average gestational age of 28 (24-29) weeks. Survival rates ranged from 48 to $100 \%$ (mean $66 \%$ ) with the largest case series not providing information [13].

\section{Shunting for LUTO}

Our combined search for LUTO retrieved 107 articles, of which 29 were found in the references of detected papers. Their number was reduced to 55 by initial screening (V.B.) and to 6 by a second evaluation (V.B. and R.D.).
Articles were excluded for: single case report (34), insufficient information (14), animal study (14), unrelated topic (13), review (12), language (11), double report (1), trial protocol (1), editorial (1) and membrane sealing (1). In total, the included publications report on 36 cases (table $1 b)$. They consist of one larger $(n=18)$ and 5 smaller case series $(n=2-6)$ with the article on 18 cases only reporting on liveborn infants [15]. No results from randomized or controlled trials were identified.

Surgery was carried out with a single-access technique and on average a maximum diameter of $2.3(1.7-2.7) \mathrm{mm}$. Mean gestational age at therapy was 21 (15-30) weeks. iPPROM occurred in an average of $31 \%(0-50 \%)$ of cases at a mean gestational age of $31(27-35)$ weeks. One article did not specify the precise gestational age at iPPROM and was excluded from this calculation. Infants were born at a mean gestational age of 33 (21-40) weeks with a mean neonatal survival rate of $91 \%$ in the 18 liveborn cases and $69 \%(17-100 \%)$ in the remaining fetuses.

\section{Prenatal Interventions for TRAP}

The combined search of the literature yielded 55 publications for TRAP, of which 31 were found via reference search. After initial screening of the abstracts by one reviewer (V.B.), 18 papers could be excluded. After critical appraisal of their full text independently by two of the authors (V.B. and R.D.) another 23 were excluded. Reasons for exclusion were: single case report (15), review (8), unrelated topic (7), double report (6), insufficient information (2), language (2) and animal study (1). The 14 articles (table 1c) left for further analysis consisted of 8 studies reporting on 2-5 cases, 2 papers on $8-11$ cases and 3 larger series with 29,51 and 60 cases, respectively. In total, data on 194 cases was obtained. No randomized or controlled trials were identified.

The modes of prenatal therapeutic interventions described were bipolar (5 studies) and monopolar (2) coagulation, cord compression (1) and ligation [(2) with or without dissection], laser of cord (3) or placenta (2), interstitial laser surgery (2), RFA (1) as well as alcohol sclerosis (1). One or 2 ports were used with a mean maximum diameter of $2.6(0.8-5) \mathrm{mm}$. Gestational age at the intervention was on average 22 (13-32) weeks and iPPROM occurred in $0-100 \%$ (mean $26 \%$ ) of cases. One article could not be included in this calculation as iPPROM rate was only specified for a 2-week interval after the intervention [16]. A precise gestational age for the time-point of iPPROM could be identified in 7 publications (mean 24, range 13-31 weeks), the remaining ones provided relative information. Infants were born at a mean gestational age 
Table 1. Systematic review of PPROM in fetal surgery

a Study characteristics for placental laser in TTTS

\begin{tabular}{|c|c|c|c|c|c|c|c|}
\hline Reference & $\mathrm{n}$ & $\varnothing, \mathrm{mm}$ & GA (Tx) & iPPROM, \% & GA (iPPROM) & GA (birth) & Survival, \% \\
\hline De Lia, 1995 [32] & 26 & 3.9 & $21(18-24)$ & 53 ( $\leq 36$ weeks) & not known & $27(19-37)$ & $53(c)$ \\
\hline Deprest, 1998 [38] & 6 & 4 & $21(19-22)$ & 50 & $29(24-34)$ & $31(24-36)$ & $67(c)$ \\
\hline Habli, 2009 [14] & 152 & 3.3 & $21 \pm 3$ & 26 & 26 & $31 \pm 5$ & $78(f)$ \\
\hline Middeldorp, 2007 [34] & 10 & 3.3 & $27(26-28)^{*}$ & 0 (<2 weeks) & - & $31(28-37)^{*}$ & $100(\mathrm{n})$ \\
\hline Middeldorp, 2007 [39] & 100 & 3.3 & $20(16-26)^{*}$ & 13 (<2 weeks) & not known & $33(18-40)^{*}$ & $70(\mathrm{n})$ \\
\hline Peiro, 2009 [40] & 148 & 3 & $21(15-25)$ & 12 & not known & 35 & $86(p)$ \\
\hline Quintero, 2001 [41] & 72 & $3(1-2)$ & $21(17-26)$ & 6 ( $\leq 3$ weeks) & not known & $32(19-41)$ & 48 \\
\hline Ruano, 2009 [33] & 19 & 2.2 & $22(19-26)$ & 11 & $29(26-32)$ & $33(26-38)$ & 53 \\
\hline Said, 2008 [42] & 10 & 2.8 & $20(18-24)^{*}$ & 30 & $2 \times<22$ weeks, $1 \times 29$ weeks & $30(22-37)^{*}$ & $65(c)$ \\
\hline Sepulveda, 2007 [43] & 33 & 3.8 & $21(17-25)^{*}$ & 15 (viable) & not known & $32(23-38)$ & $52(\mathrm{p})$ \\
\hline Ville, 1998 [37] & 132 & 3.3 & $21(15-27)^{*}$ & 10 (oligo) & not known & $32(18-40)$ & $55(\mathrm{c})$ \\
\hline Winer, 2008 [13] & 438 & 3.3 & $21(15-26)$ & 30 & $14 \% \leq 3$ weeks post $\mathrm{Tx}$ & $30 \pm 6$ & not known \\
\hline
\end{tabular}

b Study characteristics for shunting in LUTO

\begin{tabular}{lrlllrr}
\hline Reference & $\mathrm{n}$ & $\varnothing, \mathrm{mm}$ & GA (Tx) & iPPROM, \% & GA (iPPROM) & GA (birth) \\
\hline Biard, 2005 [15] & 18 & 2.5 & $22(15-29)$ & 6 (liveborns) & $1 \times 4$ days post Tx & $35(29-40)$ \\
Evans, 1991 [35] & 3 & 2.1 & $17(15-19)$ & 0 & - & $35(32-37)$ \\
Glick, 1985 [36] & 6 & 2.7 & $23(18-30)$ & 17 & 30 & $100(\mathrm{c})$ \\
Manning, 1983 [44] & 2 & 1.7 & $17(17-17)$ & 50 & 27 & $17(\mathrm{c})$ \\
Robichaux, 1991 [45] & 2 & 2.7 & $20(18-21)$ & 50 & 30 & $30(\mathrm{c})$ \\
Szaflik, 1998 [46] & 5 & 2.1 & $22(18-25)$ & 40 & $35(34-35)$ & $32(31-35)$ \\
\hline
\end{tabular}

c Study characteristics for prenatal interventions in TRAP

\begin{tabular}{|c|c|c|c|c|c|c|c|c|}
\hline Reference & $\mathrm{n}$ & $\mathrm{Tx}$ & $\varnothing, \mathrm{mm}$ & GA (Tx) & iPPROM, \% & GA (iPPROM) & GA (birth) & Survival, \% \\
\hline Deprest, 2000 [47] & 5 & $\mathrm{Bi}$ & 3 & $20(18-23)$ & 60 & not known & $37(36-39)$ & $80(c)$ \\
\hline Gallot, 2003 [48] & 3 & $\mathrm{CC}, \mathrm{Bi}$ & $5(1-2)$ & $23(22-24)$ & 33 & $\begin{array}{l}1 \times>3 \text { weeks post } \\
\mathrm{Tx}\end{array}$ & $36(30-39)$ & 100 \\
\hline $\mathrm{He}, 2010[16]$ & 4 & $\mathrm{Bi}$ & 3.9 & $23(22-25)$ & 0 ( $\leq 2$ weeks $)$ & not known & $39(38-40)$ & $50(c)$ \\
\hline Nicolini, 2001 [49] & 2 & $\mathrm{Bi}$ & 3.3 & $26(24-27)$ & 0 & - & 37 & 50 \\
\hline Hecher, 2006 [3] & 60 & Laser cord/plac, Bi & $3(1-2)$ & $19(15-25)^{*}$ & 18 & $\begin{array}{l}18 \%<34 \text { weeks } \\
\left(62 \text { days post } \mathrm{Tx}^{*}\right)\end{array}$ & $38(24-42)^{*}$ & $80(n)$ \\
\hline Ville, 1994 [50] & 4 & Laser cord & 2.7 & $23(17-28)$ & 25 & 29 & $34(29-39)$ & $100(\mathrm{c})$ \\
\hline O’Donughue, 2008 [51] & 10 & Laser abd & 1.5 & $17(13-21)$ & 10 & 13 & $30(14-39)$ & $60(\mathrm{n})$ \\
\hline Weisz, 2004 [52] & 2 & Laser abd & 1 & $22(21-23)$ & 0 & - & $35(32-37)$ & $100(\mathrm{c})$ \\
\hline Quintero, 2006 [2] & 51 & $\mathrm{CL} \pm$ cut, laser cord/plac & 3.5 & $22 \pm 3$ & 12 & not known & $30(23-40)$ & $65(n)$ \\
\hline Holmes, 2001 [8] & 11 & Mono & 1 & $21(16-24)$ & 0 & - & $38(32-42)$ & $87(\mathrm{p})$ \\
\hline Sepulveda, 2003 [9] & 2 & Mono & 1 & $23(16-29)$ & 50 & 31 & 31 & 33 \\
\hline Lee, $2007[4]$ & 29 & RFA & 1.2 & $18-24$ & 17 & not known & $35(24-40)$ & 86 \\
\hline Deprest, 1998 [53] & 3 & $\mathrm{CL}$ & $5(2)$ & $22(21-24)$ & 100 & $25(21-29)$ & $29(26-30)$ & $67(c)$ \\
\hline Sepulveda, 2004 [5] & 8 & $\mathrm{EtOH}$ & 0.8 & $25(20-32)$ & 13 & 22 & $34(23-38)$ & $63(n)$ \\
\hline
\end{tabular}

$n$ reflects the number of cases that were included from the respective publication. Data is given as mean or median $(*)$ with range or standard deviation, as known. iPPROM data is depicted until 37 weeks or for the interval/subgroup specified. If multiple ports were used, this is indicated in parentheses next to the diameter. Letters in parentheses next to survival rates represent follow-up time, if provided. $\varnothing=\mathrm{Diameter}$; $\mathrm{GA}=$ gestational age; Tx $=$ therapy; $\mathrm{iPPROM}=$ iatrogenic premature prelabor rupture of membranes; $\mathrm{f}=$ fetal; $\mathrm{p}=$ perinatal; $\mathrm{n}=\mathrm{neonatal} ; \mathrm{c}=\mathrm{childhood} ; \mathrm{Bi}=\mathrm{bipolar} ; \mathrm{Mono}=\mathrm{mo}-$ nopolar; $\mathrm{RFA}=$ radiofrequency ablation; $\mathrm{CC}=$ cord compression; $\mathrm{CL}=$ cord ligation; $\mathrm{plac}=$ placenta; $\mathrm{abd}=\mathrm{abdomen}$; EtOH $=\mathrm{alcohol}$ sclerosis

of 35 (14-42) weeks, which resulted in an average survival rate of $73 \%(33-100)$. Numbers were too small to draw conclusions about differences in outcome of the listed procedures.
Combined Statistical Analyses

A combined bivariate analysis of the included studies on TRAP, LUTO and TTTS with a total of 1,376 cases (table 2) was carried out identifying the maximum diameter of the instrument as a predictor of iPPROM rate 
Table 2. Outcome parameters and predictors in TTTS, LUTO and TRAP

\begin{tabular}{lrllll}
\hline Diagnosis & $\mathrm{n}$ & $\varnothing, \mathrm{mm}$ & iPPROM, $\%$ & GA (birth) & Survival, \% \\
\hline TTTS & 1,146 & $3.3(2.2-4), \max .2 \times$ & $27(11-53)$ & $31(18-41)$ & $66(48-100)$ \\
LUTO & 36 & $2.3(1.7-2.7)$ & $31(0-50)$ & $33(21-40)$ & $73(17-100)$ \\
TRAP & 194 & $2.6(0.8-5), \max .2 \times$ & $26(0-100)$ & $35(14-42)$ & $73(33-100)$ \\
\hline
\end{tabular}

Data is presented as mean and range. GA (iPPROM) is not depicted because of the non-representative character of the detected numbers (see table $1 \mathrm{a}-\mathrm{c}$ ). $\varnothing=$ Diameter; iPPROM = iatrogenic premature prelabor rupture of membranes; GA = gestational age.

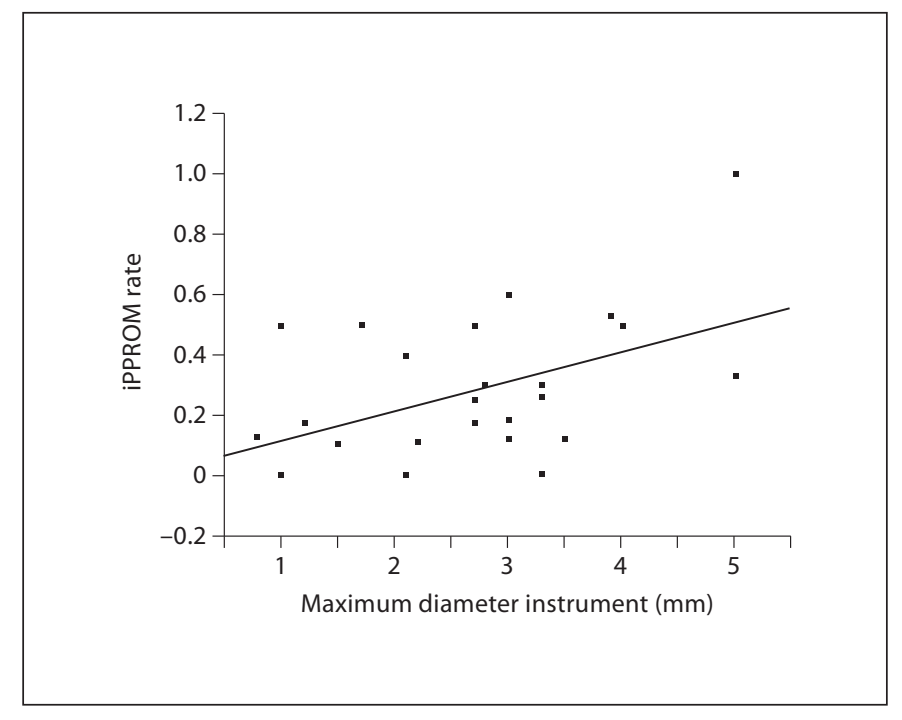

Fig. 1. Bivariate analysis of maximum diameter of the instrument and iPPROM rate. Bivariate analysis of pooled data of placental laser for TTTS, shunting for LUTO and prenatal interventions for TRAP indicates a significant correlation between the maximum diameter of the instrument and the respective iPPROM rate $\left(\mathrm{R}^{2}=0.23, \mathrm{p}=0.014\right)$.

$\left(\mathrm{R}^{2}=0.23, \mathrm{p}=0.014\right)$ (fig. 1). The diagnosis TTTS and the treatment 'placental laser' were associated with a significantly lower gestational age at birth $\left(\mathrm{R}^{2}=0.20, \mathrm{p}=0.03\right)$ compared to TRAP and its alternative (including 2 papers with placental laser among other interventions $[2,3]$ ) treatment options. Gestational age at delivery was found to predict survival $\left(\mathrm{R}^{2}=0.19, \mathrm{p}=0.015\right)$.

In the combined stepwise multivariate analysis, iPPROM rate $\left(\mathrm{R}^{2}=0.29\right)$ was explained by the maximum number of ports $(\mathrm{p}=0.21)$ as well as the maximum diameter of the instrument $(\mathrm{p}=0.05)$ used. The latter significantly influenced gestational age at birth $\left(R^{2}=0.16, p=\right.$
0.018). Survival $\left(\mathrm{R}^{2}=0.68\right)$ was explained by gestational age at birth $(\mathrm{p}=0.0044)$, gestational age at iPPROM $(\mathrm{p}=$ 0.18 ) and the maximum diameter of the instrument ( $\mathrm{p}=$ $0.016)$.

\section{Discussion}

We conducted a systematic review on iPPROM rate, gestational age at birth and fetal survival after minimally invasive surgery. In order to obtain representative information on currently performed prenatal interventions, we chose to study 3 entities that would cover different access modalities and surgical techniques: placental laser ablation for TTTS, antenatal treatment for TRAP and percutaneous bladder shunting for LUTO. Our findings indicate that $\mathrm{PPROM}$ is a frequent complication of minimally invasive fetal procedures and that the risk for membrane rupture can be predicted by the maximum diameter of the instrument used for the respective prenatal intervention. The maximum diameter is further significantly related to fetal survival as well as the gestational age at birth, which is the most important predictor of survival in our analysis. We further document considerable heterogeneity in the reporting of iPPROM data especially for TTTS cases.

For many years, spontaneous PPROM has been investigated and multiple risk factors have been identified. Major etiologic factors contributing to the pathogenesis are subclinical intrauterine infections and obstetrical characteristics of the current as well as previous pregnancies. Recent studies further attribute genetic, nutritional, behavioral and environmental factors an important role in this setting $[17,18]$. iPPROM, however, is characterized by a different underlying pathophysiology: here, the medical instrument creates the hole in the membranes, which subsequently fails to close [19]. Surgical characteristics 
that are discussed to possibly influence the incidence of iPPROM are the number and the diameter of the ports, duration and difficulty of the procedure, operator experience, membrane friction due to instrument manipulation, type of anesthesia, gestational age at intervention, number of interventions and placental location $[10,11$, 20-22]. In our review, the maximum diameter of the instrument significantly predicted the iPPROM rate and was significantly linked to gestational age at birth and fetal survival. This makes sense, as survival is highly dependent on the gestational age at birth, which again is strongly influenced by PPROM. Given the impact of gestational age at delivery onto long-term neurologic impairment, the later might be an interesting outcome for future studies to investigate in this context [23]. However, Peterson et al. [22], who evaluated 310 consecutive cases of laser surgery for TTTS in 2 centers, found no relation between a larger access cannula and the incidence of iPPROM. Diameters in this study ranged from 2.3 to 3.8 $\mathrm{mm}$, whereas in our review instruments of $0.8-5 \mathrm{~mm}$ were used. This in combination with a case number of $\sim 1,500$ might have revealed correlations in our analysis that were not obvious in the previous study. However, even in our review with pooled data of more than 1,000 cases for TTTS, statistical analyses of only this subgroup did not prove feasible. Therefore, we pooled the data of our 3 subgroups, an approach that might be criticized: substantial differences between the groups do not only exist regarding underlying diagnosis and surgical technique but also in terms of obstetrical characteristics such as singleton versus multiple pregnancies. The latter is most likely the cause for the earlier gestational age at delivery in TTTS compared to TRAP cases. We agree that the optimal scenario would be a systematic evaluation of a single fetal disorder and its subsequent treatment (alternatives). However, diagnosis and therapy were considered as variables in the regression analyses. That is why we are confident that statistical differences are not only due to subgroup-related parameters. In contrast, the highly variable settings offered us the possibility to study certain impacts on a larger scale.

The combination of data from the different subgroups was partly necessary due to a lack of consistency in reporting or in the way of reporting about iPPROM-relevant information. Interestingly, this heterogeneity was most pronounced in the TTTS group: 28,13 and $4 \%$ of identified papers had to be excluded solely for insufficient data in the TTTS, LUTO and TRAP group, respectively.

iPPROM can be a temporary condition or last for the entire course of the remaining pregnancy. In case of sub- sequent closure of the defect, this seems to be a process rather independent from histologic wound repair. Gratacos et al. [10] studied membrane samples after fetoscopic interventions. Irrespective of the time interval until birth, they detected no evidence of spontaneous membrane healing. Therefore, other closure mechanisms appear likely: first, amnion and chorion, the two membrane sheets, might slide over one another and, hence, decrease the functional defect size. Second, attachment of the membranes to the decidual layer might create functional integrity of the rupture site. This is in line with earlier publications, pointing out the connection of postsurgical chorion-amnion separation and an increased risk of iPPROM [24-26]. As a consequence, an oblique puncture technique was suggested with some success in vitro [27].

In view of the frequency and severity of iPPROM, several teams have attempted to develop strategies to seal membrane defects at the time of the fetal intervention or at the time of clinically overt fluid leakage [28-31]. None of these strategies has, however, become a standard of care.

Our review was performed according to current Cochrane guidelines: search as well as inclusion and exclusion criteria were specified ahead and any change to the original protocol was noted. Two reviewers evaluated the detected articles independently of each other and disagreements had to be resolved by consensus. Our pooled statistical analysis is well powered with the evaluation of almost 1,500 cases and 10 different treatment modalities.

We certainly acknowledge that there are also limitations to our approach. For example, the adaptation of our original protocol may be subject to criticism. Yet, a change of the strict rules defining a systematic review might very well prove reasonable during its conduction, something that is also taken into consideration in the Cochrane guidelines (www.cochrane-handbook.org). In addition, the limitation to English language papers may be a barrier. However, as English is the lingua franca of science, we are confident that the most important publications on the defined topics were detected. Surprisingly, our overall search only yielded 2 randomized controlled trials $[1,12]$, both of which were excluded in favor of bigger and more recent publications of the respective teams $[13,14]$. This leaves our review with case series alone that - given publication bias and despite the exclusion of single case reports - might present a more optimistic version of the clinical reality.

Another drawback of our review is the missing correction for certain clinical data: several TTTS series comprised higher multiple pregnancies $[13,14]$ and some contain cases with septostomy either intended or as an unde- 
sired side effect of fetal surgery $[2,32]$. Also the inclusion criteria for treatment were different depending on the center: some treated early, some only advanced stages: TTTS I patients e.g. would only have received laser ablation in distinct centers $[14,33]$. Further, polyhydramnios was sometimes a requirement for TRAP therapy $[2,9]$. Moreover, gestational age at treatment varied considerably: Middeldorp et al. [34] conducted a study on laser treatment for TTTS after 26 weeks, a gestational age that was not treated by fetal intervention in any of the other centers. In the case of LUTO, doctors first had to learn about the deleterious effect of oligohydramnios on lung development, before late shunting was abandoned, which leaves earlier trials with a worse outcome $[35,36]$. Further, there is variation of the administration of antibiotics $[14,37]$ and the institutional protocol for a short cervix [37]. All of these factors might clearly have influenced outcome in terms of PPROM, preterm birth and subsequent survival. However, information on these items is so scarce that a correction for them was not feasible. Data was sometimes also limited regarding the patient cohort reported on, for example Biard et al. [15] only describe liveborns. These numbers were marked accordingly and have to be appraised critically by the reader. Another potential confounder is the follow-up time: some children were evaluated only in utero, others were followed until several years into their childhood [14, 38]. Again, data presentation did not allow to correct for these highly different intervals. However, as this is the inherent nature of clinical reports like the ones we studied and given a higher likelihood for early rather than late adverse events in the setting of fetal pathology, we feel the overall impact on our conclusions is limited. The diameter of the instrument was the strongest predictor of iPPROM among the parameters we analyzed. This result does not exclude other factors, not available for analysis (e.g. duration of the respective procedure), to have a strong influence.

Any type of study in the field of fetal surgery is challenged by relatively small patient numbers. This in com- bination with differences due to the respective center, diagnosis and procedure might explain, why some potential influencing factors of iPPROM are identified as relevant in one publication [21] and do not seem to play a role in other studies [20]. Hence, beside multicenter trials, systematic reviews and meta-analyses are of great importance to obtain a big enough dataset for meaningful analysis. In order to be able to compare the data from different institutions, however, certain standards of data publication are required.

We therefore suggest (1) to define what is considered as PPROM in the respective publication, (2) to specify the method by which the diagnosis of PPROM was made, (3) to give information on all cases with PPROM until 37 weeks of pregnancy, (4) to provide a gestational age at PPROM, and (5) to also report subsequent closure of the defect. This way the entire risk of PPROM after fetal interventions becomes apparent - in its life-threatening early as well as in its less severe late onset variant. A more complete dataset on iPPROM rate on the other hand will allow for in-depth investigation of risk factors, thus helping to prevent iPPROM. Successful reduction of iPPROM numbers is a crucial step for further advancement of fetal surgery as long as no treatment exists for this major complication.

\section{Acknowledgements}

The European Commission supports fetoscopy in Europe through different funding mechanisms (EuroSTEC, 6th Framework, LSHC-CT-2006-037409; Marie Curie Early Stage Research Training MEST CT2005 019707), and provided the doctoral grants for V.B. and L.G. V.B. is further supported by a Marie Curie Reintegration Grant (PERG07-GA-2010-268330; FP7-PEOPLE-2010-RG). The Flemish Regional Government (IWT/070715) supports our local fetoscopy program for Congenital Diaphragmatic Hernia. R.D. is a senior postdoctoral researcher (FWO 2010-15).

\section{References}

Systematic Review of PPROM in Fetal Surgery
1 Senat MV, Deprest J, Boulvain M, Paupe A, Winer N, Ville Y: Endoscopic laser surgery versus serial amnioreduction for severe twin-to-twin transfusion syndrome. $\mathrm{N}$ Engl J Med 2004;351:136-144.

- 2 Quintero RA, Chmait RH, Murakoshi T, Pankrac Z, Swiatkowska M, Bornick PW, Allen $\mathrm{MH}$ : Surgical management of twin reversed arterial perfusion sequence. Am J Obstet Gynecol 2006;194:982-991.
-3 Hecher K, Lewi L, Gratacos E, Huber A, Ville Y, Deprest J: Twin reversed arterial perfusion: fetoscopic laser coagulation of placental anastomoses or the umbilical cord. Ultrasound Obstet Gynecol 2006;28:688-691.

-4 Lee H, Wagner AJ, Sy E, Ball R, Feldstein VA, Goldstein RB, Farmer DL: Efficacy of radiofrequency ablation for twin-reversed arterial perfusion sequence. Am J Obstet Gynecol 2007;196:459 e451-e454. 
$\checkmark 5$ Sepulveda W, Corral E, Aiello H, Otano L, Paredes R, Escobar MF, Heredia F, Quiroz V: Intrafetal alcohol chemosclerosis of acardiac twins: a multicenter experience. Fetal Diagn Ther 2004; 19:448-452.

6 Sepulveda W, Sebire NJ: Acardiac twin: too many invasive treatment options - the problem and not the solution. Ultrasound Obstet Gynecol 2004;24:387-389.

-7 Van der Wildt B, Luks FI, Steegers EA, Deprest JA, Peers KH: Absence of electrical uterine activity after endoscopic access for fetal surgery in the rhesus monkey. Eur J Obstet Gynecol Reprod Biol 1995;58:213-214.

$>8$ Holmes A, Jauniaux E, Rodeck C: Monopolar thermocoagulation in acardiac twinning. BJOG 2001;108:1000-1002.

-9 Sepulveda W, Corral E, Gutierrez J: A simple device for vascular occlusion of acardiac twins. Ultrasound Obstet Gynecol 2003;21: 386-388.

-10 Gratacos E, Sanin-Blair J, Lewi L, Toran N, Verbist G, Cabero L, Deprest J: A histological study of fetoscopic membrane defects to document membrane healing. Placenta 2006;27: 452-456.

11 Chang J, Tracy TF Jr, Carr SR, Sorrells DL Jr, Luks FI: Port insertion and removal techniques to minimize premature rupture of the membranes in endoscopic fetal surgery. J Pediatr Surg 2006;41:905-909.

$\checkmark 12$ Crombleholme TM, Shera D, Lee H, Johnson M, D’Alton M, Porter F, Chyu J, Silver R, Abuhamad A, Saade G, Shields L, Kauffman D, Stone J, Albanese CT, Bahado-Singh R, Ball RH, Bilaniuk L, Coleman B, Farmer D, Feldstein V, Harrison MR, Hedrick H, Livingston J, Lorenz RP, Miller DA, Norton ME, Polzin WJ, Robinson JN, Rychik J, Sandberg PL, Seri I, Simon E, Simpson LL, Yedigarova L, Wilson RD, Young B: A prospective, randomized, multicenter trial of amnioreduction vs. selective fetoscopic laser photocoagulation for the treatment of severe twin-twin transfusion syndrome. Am J Obstet Gynecol 2007;197:396 e391-e399.

-13 Winer N, Salomon LJ, Essaoui M, Nasr B, Bernard JP, Ville Y: Pseudoamniotic band syndrome: a rare complication of monochorionic twins with fetofetal transfusion syndrome treated by laser coagulation. Am J Obstet Gynecol 2008;198:393 e391-395.

14 Habli M, Bombrys A, Lewis D, Lim FY, Polzin W, Maxwell R, Crombleholme T: Incidence of complications in twin-twin transfusion syndrome after selective fetoscopic laser photocoagulation: a single-center experience. Am J Obstet Gynecol 2009;201:417 e411-417.

-15 Biard JM, Johnson MP, Carr MC, Wilson RD, Hedrick HL, Pavlock C, Adzick NS: Long-term outcomes in children treated by prenatal vesicoamniotic shunting for lower urinary tract obstruction. Obstet Gynecol 2005; 106:503-508.
16 He ZM, Fang Q, Yang YZ, Luo YM, Chen JH, Chen YZ, Zhou Y, Chen ML: Fetal reduction by bipolar cord coagulation in managing complicated monochorionic multiple pregnancies: preliminary experience in China. Chin Med J (Engl) 2010;123:549-554.

17 Devlieger R, Millar LK, Bryant-Greenwood G, Lewi L, Deprest JA: Fetal membrane healing after spontaneous and iatrogenic membrane rupture: a review of current evidence. Am J Obstet Gynecol 2006;195:1512-1520.

18 Mingione MJ, Pressman EK, Woods JR: Prevention of PPROM: current and future strategies. J Matern Fetal Neonatal Med 2006;19: 783-789.

19 Devlieger R, Gratacos E, Wu J, Verbist L, Pijnenborg R, Deprest JA: An organ culture for in vitro evaluation of fetal membrane healing capacity. Eur J Obstet Gynecol Reprod Biol 2000;92:145-150.

20 Jani JC, Nicolaides KH, Gratacos E, Valencia CM, Done E, Martinez JM, Gucciardo L, Cruz R, Deprest JA: Severe diaphragmatic hernia treated by fetal endoscopic tracheal occlusion. Ultrasound Obstet Gynecol 2009; 34:304-310.

21 Lewi L, Gratacos E, Ortibus E, Van Schoubroeck D, Carreras E, Higueras T, Perapoch J, Deprest J: Pregnancy and infant outcome of 80 consecutive cord coagulations in complicated monochorionic multiple pregnancies. Am J Obstet Gynecol 2006;194:782-789.

22 Petersen S, Doné E, Gardener G, Lewi L, van Schoubroeck D, Devlieger R, Chan FY, de Catte L, Deprest J: Rate of amniorrhexis is not influenced by fetoscopic cannula diameter: 19th World Congress on Ultrasound in Obstetrics and Gynecology, Hamburg 2009.

23 Lopriore E, Ortibus E, Acosta-Rojas R, Le Cessie S, Middeldorp JM, Oepkes D, Gratacos E, Vandenbussche FP, Deprest J, Walther FJ, Lewi L: Risk factors for neurodevelopment impairment in twin-twin transfusion syndrome treated with fetoscopic laser surgery. Obstet Gynecol 2009;113:361-366.

-24 Sydorak RM, Hirose S, Sandberg PL, Filly RA, Harrison MR, Farmer DL, Albanese CT: Chorioamniotic membrane separation following fetal surgery. J Perinatol 2002;22: 407-410.

25 Devlieger R, Van Mieghem T, Lewi L, De Catte L, Van Schoubroeck D, Deprest J: Chorionic membrane separation following fetoscopy. A role for collagen plugging of the fetoscopic access site? Commentary on Chang et al: Transient chorioamniotic membrane separation after fetoscope guide laser therapy for twin-twin transfusion syndrome: a case report (Fetal Diagn Ther 2007;22:180-182). Fetal Diagn Ther 2008;23:87-88, author reply 88.

26 Papanna R, Mann LK, Johnson A, SangiHaghpeykar H, Moise KJ Jr: Chorioamnion separation as a risk for preterm premature rupture of membranes after laser therapy for twin-twin transfusion syndrome. Obstet Gynecol 2010;115:771-776.
27 Gratacos E, Devlieger R, Decaluwe H, Wu J, Nicolini U, Deprest JA: Is the angle of needle insertion influencing the created defect in human fetal membranes? Evaluation of the agreement between specialists' opinions and ex vivo observations. Am J Obstet Gynecol 2000;182:646-649.

-28 Lewi L, Van Schoubroeck D, Van Ranst M, Bries G, Emonds MP, Arabin B, Welch R, Deprest J: Successful patching of iatrogenic rupture of the fetal membranes. Placenta 2004;25:352-356

29 Papanna R, Molina S, Moise KY, Moise KJ Jr, Johnson A: Chorioamnion plugging and the risk of preterm premature rupture of membranes after laser surgery in twin-twin transfusion syndrome. Ultrasound Obstet Gynecol 2010;35:337-343.

>30 Ochsenbein-Kolble N, Jani J, Lewi L, Verbist G, Vercruysse L, Portmann-Lanz B, Marquardt K, Zimmermann R, Deprest J: Enhancing sealing of fetal membrane defects using tissue engineered native amniotic scaffolds in the rabbit model. Am J Obstet Gynecol 2007;196:263 e261-e267.

-31 Quintero RA, Kontopoulos EV, Chmait R, Bornick PW, Allen M: Management of twintwin transfusion syndrome in pregnancies with iatrogenic detachment of membranes following therapeutic amniocentesis and the role of interim amniopatch. Ultrasound $\mathrm{Ob}$ stet Gynecol 2005;26:628-633.

32 De Lia JE, Kuhlmann RS, Harstad TW, Cruikshank DP: Fetoscopic laser ablation of placental vessels in severe previable twin-twin transfusion syndrome. Am J Obstet Gynecol 1995;172:1202-1211.

33 Ruano R, Brizot Mde L, Liao AW, Zugaib M: Selective fetoscopic laser photocoagulation of superficial placental anastomoses for the treatment of severe twin-twin transfusion syndrome. Clinics (São Paulo) 2009;64:9196.

34 Middeldorp JM, Lopriore E, Sueters M, Klumper FJ, Kanhai HH, Vandenbussche FP, Oepkes D: Twin-to-twin transfusion syndrome after 26 weeks of gestation: is there a role for fetoscopic laser surgery? BJOG 2007; 114:694-698.

-35 Evans MI, Sacks AJ, Johnson MP, Robichaux AG 3rd, May M, Moghissi KS: Sequential invasive assessment of fetal renal function and the intrauterine treatment of fetal obstructive uropathies. Obstet Gynecol 1991;77: 545-550

36 Glick PL, Harrison MR, Golbus MS, Adzick NS, Filly RA, Callen PW, Mahony BS, Anderson RL, deLorimier AA: Management of the fetus with congenital hydronephrosis. II. Prognostic criteria and selection for treatment. J Pediatr Surg 1985;20:376-387.

-37 Ville Y, Hecher K, Gagnon A, Sebire N, Hyett J, Nicolaides K: Endoscopic laser coagulation in the management of severe twin-to-twin transfusion syndrome. Br J Obstet Gynaecol 1998; 105:446-453. 
-38 Deprest JA, Van Schoubroeck D, Van Ballaer PP, Flageole H, Van Assche FA, Vandenberghe K: Alternative technique for Nd:YAG laser coagulation in twin-to-twin transfusion syndrome with anterior placenta. Ultrasound Obstet Gynecol 1998;11:347-352.

-39 Middeldorp JM, Sueters M, Lopriore E, Klumper FJ, Oepkes D, Devlieger R, Kanhai $\mathrm{HH}$, Vandenbussche FP: Fetoscopic laser surgery in 100 pregnancies with severe twinto-twin transfusion syndrome in The Netherlands. Fetal Diagn Ther 2007;22:190-194.

-40 Peiro JL, Carreras E, Guillen G, Arevalo S, Sanchez-Duran MA, Higueras T, Castillo F, Marhuenda C, Lloret J, Martinez-Ibanez V: Therapeutic indications of fetoscopy: a 5-year institutional experience. J Laparoendosc Adv Surg Tech A 2009;19:229-236.

-41 Quintero RA, Bornick PW, Allen MH, Johson PK: Selective laser photocoagulation of communicating vessels in severe twin-twin transfusion syndrome in women with an anterior placenta. Obstet Gynecol 2001;97:477481.

-42 Said S, Flood K, Breathnach F, Fleming A, Kinsella CB, Geary M, Malone FD: Fetoscopic laser treatment of twin-to-twin transfusion syndrome. Ir Med J 2008;101:191-193.
43 Sepulveda W, Wong AE, Dezerega V, Devoto JC, Alcalde JL: Endoscopic laser surgery in severe second-trimester twin-twin transfusion syndrome: a three-year experience from a Latin American center. Prenat Diagn 2007; 27:1033-1038.

44 Manning FA, Harman CR, Lange IR, Brown R, Decter A, MacDonald N: Antepartum chronic fetal vesicoamniotic shunts for obstructive uropathy: a report of two cases. Am J Obstet Gynecol 1983;145:819-822.

45 Robichaux AG 3rd, Mandell J, Greene MF, Benacerraf BR, Evans MI: Fetal abdominal wall defect: a new complication of vesicoamniotic shunting. Fetal Diagn Ther 1991;6:1113.

46 Szaflik K, Kozarzewski M, Adamczewski D: Fetal bladder catheterization in severe obstructive uropathy before the 24th week of pregnancy. Fetal Diagn Ther 1998;13:133135.

47 Deprest JA, Audibert F, Van Schoubroeck D, Hecher K, Mahieu-Caputo D: Bipolar coagulation of the umbilical cord in complicated monochorionic twin pregnancy. Am J Obstet Gynecol 2000;182:340-345.
48 Gallot D, Laurichesse H, Lemery D: Selective feticide in monochorionic twin pregnancies by ultrasound-guided umbilical cord occlusion. Ultrasound Obstet Gynecol 2003;22: 484-488.

49 Nicolini U, Poblete A, Boschetto C, Bonati F, Roberts A: Complicated monochorionic twin pregnancies: experience with bipolar cord coagulation. Am J Obstet Gynecol 2001; 185:703-707.

-50 Ville Y, Hyett JA, Vandenbussche FP, Nicolaides $\mathrm{KH}$ : Endoscopic laser coagulation of umbilical cord vessels in twin reversed arterial perfusion sequence. Ultrasound Obstet Gynecol 1994;4:396-398.

-51 O’Donoghue K, Barigye O, Pasquini L, Chappell L, Wimalasundera RC, Fisk NM: Interstitial laser therapy for fetal reduction in monochorionic multiple pregnancy: loss rate and association with aplasia cutis congenita. Prenat Diagn 2008;28:535-543.

52 Weisz B, Peltz R, Chayen B, Oren M, Zalel Y, Achiron R, Lipitz S: Tailored management of twin reversed arterial perfusion sequence. Ultrasound Obstet Gynecol 2004;23:451455.

53 Deprest JA, Van Ballaer PP, Evrard VA, Peers KH, Spitz B, Steegers EA, Vandenberghe K: Experience with fetoscopic cord ligation. Eur J Obstet Gynecol Reprod Biol 1998;81:157164. 